\title{
Disseminated Coccidioidomycosis Refractory to Multi-Drug Therapies in an Immunocompetent Patient
}

\author{
Razi Syed Haq ${ }^{1,3 *}$, Farbod Farmand ${ }^{1}$, Sharon Wang ${ }^{2}$, Anthony Rajasingham ${ }^{1}$, Lloyd Muzangwa ${ }^{3}$ and Samuel \\ Beaujean $^{3}$
}

${ }^{1}$ Department of Internal Medicine, Arrowhead Regional Medical Center, USA

${ }^{2}$ Department of Infectious Disease, Arrowhead Regional Medical Center, USA

${ }^{3}$ St George's University School of Medicine, West Indies

Submission: June 28, 2019; Published: August 09, 2019

*Corresponding author: Razi Syed Haq, Department of Internal Medicine, Arrowhead Regional Medical Center, Colton, CA, USA

\begin{abstract}
Coccidioidomycosis is a fungal infection caused by Coccidioides immitis which thrives in dry desert climates such as the southwestern United States. Disseminated coccidioidomycosis is often seen in patients with an underlying immunodeficiency. Historically there have been few reported cases of opportunistic infections in the setting of low CD4+ count and no evidence of HIV infection. Coccidioidomycosis typically presents asymptomatically or as a minor respiratory illness in immunocompetent individuals. We report a case of disseminated coccidioidomycosis in a patient who is otherwise immunocompetent. Patient is a 24-year-old Hispanic male who presented with severe headache, fever, body pain, vomiting, and diarrhea for approximately 14 days. On admission, patient's CD4+ count was 91 and CSF culture grew Coccidioides immitis, for which patient was started on fluconazole. Patient's hospital stay was complicated by multiple bouts of altered mental status, fevers, hyponatremia, hydrocephalus, neurogenic bladder, new-onset Mobitz II heart arrhythmia, urinary tract infection, and sepsis. MRI studies revealed abnormal enhancement of leptomeninges, bilateral basal ganglia, thecal sac and cauda equina fibers, signifying disseminated disease. Patient's serum Coccidioides complement fixation (IgG) titers remained elevated despite aggressive therapy with IV amphotericin B. After a total of six months of hospitalization, including treatment with intrathecal amphotericin B, patient's last CD4+ count was 549, CSF IgG titers achieved $<1: 1$ and final CSF analysis revealed glucose, protein and WBCs within normal limits. Patient was stable for transfer to a tertiary care clinic for life-long management with intrathecal amphotericin B.
\end{abstract}

Keywords: Disseminated; Coccidioidomycosis; Immunocompetent; Coccidioides immitis; Multi-drug resistance

\section{Introduction}

Coccidioidomycosis is a fungal infection caused by Coccidioides immitis, a dimorphic fungus that lives in the soil of dry desert climates of the southwestern United States, particularly Arizona, California and Mexico [1]. This fungus exists in the soil as arthroconidia which becomes airborne by wind or earthquakes. Inhaled arthroconidia transform into spherules, which release endospores and cause inflammatory changes within the lung [2]. The spherule-endospore phase of Coccidioides immitis is not infectious; thus, it is not transmissible from human-tohuman [3]. Coccidioidomycosis can remain asymptomatic, manifest as a mild influenza-like illness, or present as pneumonia with pulmonary consolidation on chest $\mathrm{x}$-ray. Disseminated coccidioidomycosis refers to infection that has spread to sites other than the lung; less than $5 \%$ of patients develop extra-pulmonary symptoms such as skin lesions and bone pain and are typically those immunocompromised [4]. Meningitis is the most serious complication of disseminated coccidioidomycosis and is lethal for $95 \%$ of untreated patients within two years of acquisition [5]. Method

This case report was formulated from the review of patient's medical records as well as observation on the inpatient ward.

\section{Case Presentation}

A 24-year-old Hispanic male presented to our ER complaining of severe headache for approximately 14 days. The 


\section{Open Access Journal of Neurology \& Neurosurgery}

patient described the headache as constant pressure involving the entire head, rated $10 / 10$. The patient endorsed associated symptoms of fever $101 \stackrel{\circ}{\circ}$, neck pain, back pain, nausea, vomiting, and diarrhea. The patient denied vision abnormalities, altered mental status, focal weakness, chest pain/pressure, cough, trouble breathing, abdominal pain, sick contacts or history of recent travel.

The patient initially presented at another hospital three days prior. Records of CSF analysis showed low glucose, elevated protein, gram stain negative, and culture negative. Notably, patient's CD4+ count was 270 and HIV ab was negative. Patient was diagnosed with bacterial meningitis and discharged two days later with cefuroxime and ibuprofen. Patient subsequently presented to our ER for unresolved symptoms. Chest x-ray and head CT without contrast were unremarkable. Serum sodium demonstrated hyponatremia and CSF analysis demonstrated low glucose, elevated protein, and elevated WBCs. Patient was treated for suspected bacterial meningitis with ceftriaxone, rifampin (vancomycin allergy), dexamethasone and electrolyte repletion with plan to follow-up CSF culture results from the other hospital. Other hospital records arrived three days later which revealed serum Coccidioides ab positive at 1511 with negative CSF culture. Current labs revealed CD4+ count of 91, and CSF culture was positive for Coccidioides immitis. Based on these results, fluconazole $1000 \mathrm{mg}$ daily was initiated, and ceftriaxone and rifampin were discontinued.
During this time, patient was upgraded to the ICU for worsening symptomatic hyponatremia. Patient expressed incoherent speech and could not follow commands. Patient had a right femoral line placed and an external lumbar drain placed by neurosurgery during which patient exhibited status epilepticus. Patient was intubated and started on propofol drip and levetiracetam. Patient also demonstrated erratic movement of the right arm; however, EEG was negative for any epileptic potentials. Later that night, patient developed a fever of $105 \stackrel{\circ}{\circ}$ and was started on broad-spectrum antibiotics linezolid and piperacillin/tazobactam. IV amphotericin B was added on at 370 mg daily, and fluconazole was increased to $1200 \mathrm{mg}$ daily.

Brain MRI revealed multifocal bilateral diffusion abnormality consistent with multifocal infarcts involving bilateral basal ganglia, as shown in Figure 1A \& B. Coccidioidomycosis-induced vasculitis could not be ruled out, so dexamethasone was maintained. Blood cultures were negative and trans-esophageal echocardiogram showed no evidence for any cardiac source of embolism. Two weeks later, patient developed acute kidney injury which prompted an adjustment of fluconazole back to $1000 \mathrm{mg}$, IV amphotericin B from daily to three times per week, and a taper of dexamethasone which effectively resolved the AKI. However, fluconazole was switched to IV voriconazole 400 $\mathrm{mg}$ twice daily as a result of acute liver injury.
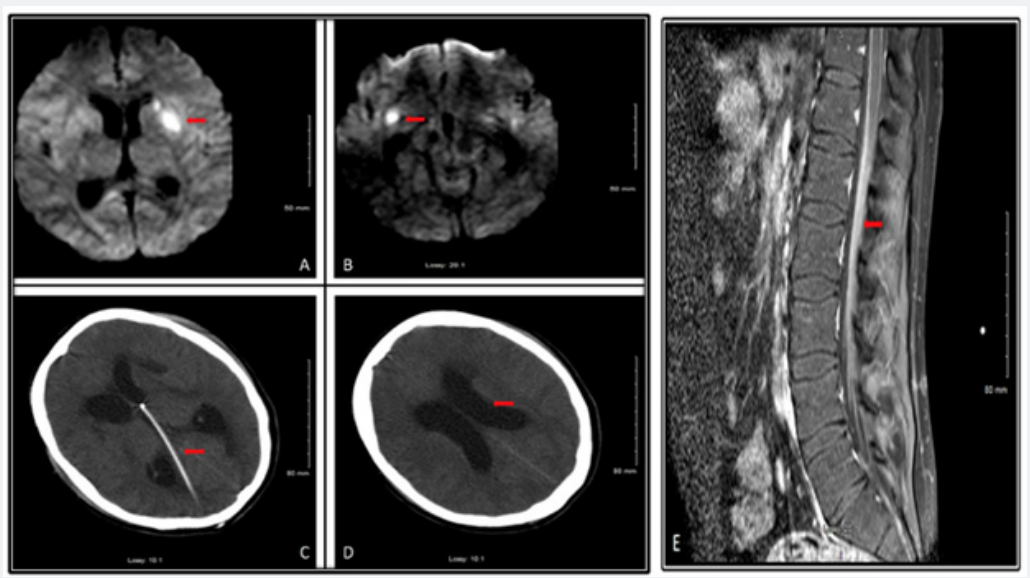

Figure 1: 24-year-old Hispanic male complaining of severe headache for approximately 14 days presents to the ER. Brain MRI demonstrates basal ganglia infarction bilaterally (A,B). CT head demonstrates hydrocephalus (C) and VP shunt (D). Lumbar spine MRI demonstrates increased signal intensity along cauda equina, signifying disseminated disease.

The patient's hospital stay was also complicated by the development of acute urinary retention. MRI studies revealed abnormal enhancement along the thecal sac and cauda equina fibers, as shown in Figure 1E, likely signifying disseminated coccidioidomycosis refractory to multi-antifungal therapy. Other clinical sequelae included hydrocephalus, which was managed by neurosurgery via ventriculoperitoneal shunt, as shown in Figure 1C \& D, and new-onset Mobitz II heart arrhythmia, for which cardiology recommended holding pacemaker implantation until systemic infection is eradicated. Patient's health condition improved and deteriorated throughout hospital stay with multiple episodes of fevers, headache, vomiting, depression, and altered mental status secondary to encephalopathy from disseminated disease. Patient's clinical symptoms stabilized after three months of hospitalization and patient was instructed to follow-up at a tertiary care center.

However, during a routine clinic visit eleven weeks later, patient was referred to the ER for elevated liver enzymes, right 
upper quadrant abdominal pain rated 5 out of 10 , persistent nausea and non-bloody/non-bilious vomiting. Patient's labs revealed negative hepatitis panel, elevated INR, and negative HIV ab. Of significance, patient's CD4+ count was 549, indicating immunocompetence. Lumbar puncture revealed low glucose, elevated protein, and positive CSF Coccidioides IgG titers. Trans jugular liver biopsy revealed severe steatohepatitis. Patient was given Vitamin $\mathrm{K}$ and restarted on IV amphotericin B. As druginduced hepatotoxicity was suspected, patient was discontinued from fluconazole and voriconazole. Patient's serum Coccidioides IgG titers remained elevated despite multi-drug therapy, signifying refractory disease. Patient was started on oral is a vuconazole $372 \mathrm{mg}$ along with IV amphotericin B.

An Ommaya reservoir was placed by neurosurgery per infectious disease recommendations in order to administer intrathecal amphotericin B. Two weeks post-op, patient received the first dose of intrathecal amphotericin $0.1 \mathrm{mg}$ and methylprednisolone with the goal of increasing the dose by 0.1 mg per week as tolerated. IV amphotericin B was discontinued. After a total of six months of hospitalization, patient's final CD4+ count was 549, Coccidioides CSF IgG titers achieved < 1:1. Patient was stable for transfer to a Coccidioides tertiary care clinic for life-long management with intrathecal amphotericin B.

\section{Discussion}

Our patient was HIV-negative and had a CD4+ count of less than 300 on more than one occasion after having acquired coccidioidomycosis, hinting at a possible virulent mechanism of Coccidioidomycosis suppressing the proliferation of CD4+ cells, resulting in Idiopathic CD4 Lymphopenia. A similar case is identified wherein a patient with disseminated coccidioidomycosis displayed persistently decreased CD4+ counts in the absence of other possible etiologies [6]. However, CD4+ counts may not paint the entire picture. Ampel et al. [7] found that symptomatic coccidioidomycosis was associated with low serum mannose-binding lectin (MBL) levels [7]. Mannosebinding lectins play a critical role in the immune response by attaching to pathogens such as bacteria, viruses, or fungi and activating the complement system for pathogenic destruction [8].

The treatment of coccidioidomycosis typically begins with oral fluconazole, 400-1000 mg per day. Voriconazole has been reported to be successful in some patients who fail or cannot tolerate fluconazole. IV amphotericin B can be initiated in the case of azole failure or disseminated disease. For refractory disease, intrathecal amphotericin B in addition to a life-long azole is recommended. Disease complicated by hydrocephalus nearly always requires a shunt for decompression [9].

\section{Conclusion}

Disseminated coccidioidomycosis is a rare presentation of a rare infection that was once thought to affect only immunocompromised individuals. We now understand that this is not the case. Coccidioidomycosis may exert its virulence by suppressing the production of CD4+ cells, mannose-binding lectins or another mechanism; thereby, compromising an otherwise normal-functioning immune system. This extremely rare case warrants further investigation into the virulence of Coccidioides immitis that leaves patients susceptible to this debilitating disease.

\section{Acknowledgement}

We would like to thank the Departments of Internal Medicine, Family Medicine, and Infectious Disease for their tireless management of this patient. We would also like to thank all the other departments who were consulted regarding this case. Finally, we would like to thank the patient for allowing us to participate in his care.

\section{References}

1. Brown J, Benedict K, Park BJ, Thompson GR (2013) Coccidioidomycosis: epidemiology. Clin Epidemiol 5:185-197.

2. Lee CH, Wilcox L, Chorneyko K, McIvor A (2008) Coccidioides immitis: two cases of misidentified mycosis. Can Respir J 15(7): 377-379.

3. Gaidici A, Saubolle MA (2009) Transmission of Coccidioidomycosis to a Human via a Cat Bite. J Clin Microbiol 47 (2): 505-506.

4. Chuang A, Thomas R, Hoffman RS (2005) Disseminated coccidioidomycosis in an immunocompetent person living in New York City. J Urban Health 82(2): 339-345.

5. Seehusen DA, Reeves M, Fomin D (2003) Cerebrospinal Fluid Analysis. Am Fam Physician 68(6):1103-1109.

6. Mathur A, Carr T (2015) A Novel Case of Idiopathic CD4 Lymphopenia Presenting with Disseminated Coccidioidomycosis 135(2): AB181.

7. Ampel NM, Dionne SO, Giblin A, Podany AB, Galgiani J (2009) MannoseBinding Lectin Serum Levels are Low in Persons with Clinically Active Coccidioidomycosis. Mycopathologia 167(4): 173-180.

8. Mannose-binding lectin deficiency - Genetics Home Reference - NIH US National Library of Medicine.

9. Galgiani JN, Ampel NM, Blair JE, Catanzaro A, Geertsma F, et al. (2016) Infectious Diseases Society of America (IDSA) Clinical Practice Guideline for the Treatment of Coccidioidomycosis. Clin Infect Dis 63(6): e112-46. 
This work is licensed under Creative Commons Attribution 4.0 Licens

DOI: 10.19080/OAJNN.2019.11.555809
Your next submission with Juniper Publishers will reach you the below assets

- Quality Editorial service

- Swift Peer Review

- Reprints availability

- E-prints Service

- Manuscript Podcast for convenient understanding

- Global attainment for your research

- Manuscript accessibility in different formats ( Pdf, E-pub, Full Text, Audio)

- Unceasing customer service

Track the below URL for one-step submission https://juniperpublishers.com/online-submission.php 